\title{
Percepção dos adolescentes acerca do suicídio
}

\author{
Adolescents' Perceptions of Suicide \\ Percepciones de los adolescentes sobre el suicídio
}

Recebido: 09/08/2021 | Revisado: 18/18/2021 | Aceito: 12/10/2021 | Publicado: 14/10/2021

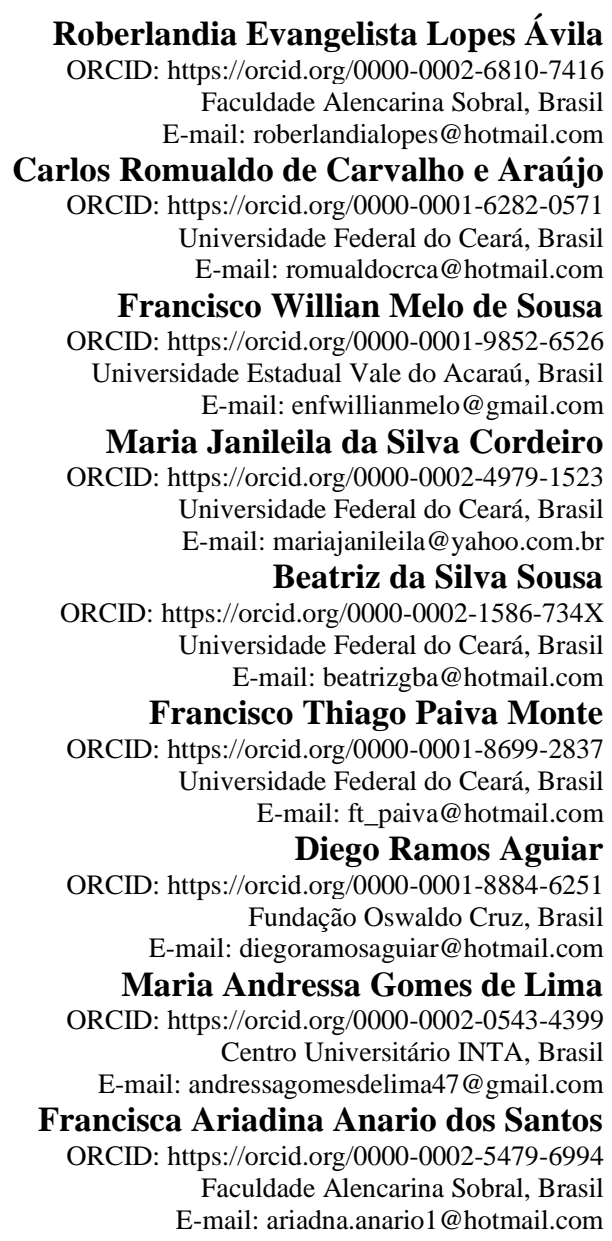

\begin{abstract}
Resumo
O estudo objetiva conhecer a percepção dos adolescentes acerca do suicídio em uma Escola de ensino médio em SobralCeará, Brasil. Estudo exploratório descritivo com abordagem qualitativa realizado com 30 adolescentes, do $3^{\circ}$ ano do ensino médio em uma Escola município do interior do Ceará em setembro de 2018. A pesquisa se valeu de uma entrevista semiestruturada com as seguintes perguntas: O que você entende por suicídio? Você consegue identificar uma ideia suicida? Se sim descreva. Se você conhecesse alguém que estaria pensando em cometer suicídio o que você faria para tentar ajuda-lo? A análise das informações seguiu os preceitos de Minayo (2010). Evidenciou-se, que os adolescentes possuem conhecimento sobre a temática, assim como dispõe de algumas estratégias de apoio diante do suicídio. A maioria dos adolescentes relatou que o primeiro contato com o tema suicídio foi na escola, bem como conheciam ou conviviam com algum indivíduo que já cometeu suicídio com isso. Salienta-se que este trabalho possa estimular tanto os familiares, como no campo da assistência a orientar os adolescentes sobre o suicídio e sobre a quebra do tabu que esse tema possui. Reforça-se também a importância de realizar novas pesquisas sobre o tema, para ampliar novas estratégias de intervenções junto a este grupo e as instituições de saúde e educação envolvidos.
\end{abstract}

Palavras-chave: Percepção do suicídio; Fatores de riscos; Prevenção; Adolescentes.

\section{Abstract}

The study aims to understand the perception of adolescents about suicide in a high school in Sobral-Ceará, Brazil. Descriptive exploratory study with a qualitative approach conducted with 30 teenagers, from the 3rd year of high school at a school in the interior of Ceará, in September 2018. The research used a semi-structured interview with the 
following questions: What do you understand by suicide? Can you identify a suicidal idea? If yes, describe. If you knew someone who would be thinking about committing suicide what would you do to try to help them? The analysis of the information followed the precepts of Minayo (2010). It was evident that adolescents have knowledge on the subject, as well as some support strategies in the face of suicide. Most adolescents reported that their first contact with the topic of suicide was at school, and that they knew or lived with someone who had already committed suicide with it. It is noteworthy that this work can encourage both family members and in the field of care to guide adolescents about suicide and about breaking the taboo that this topic has. It also reinforces the importance of conducting further research on the subject, to expand new intervention strategies with this group and the health and education institutions involved.

Keywords: Suicide perception; Risk factors; Prevention; Teenagers.

\section{Resumen}

El estudio tiene como objetivo comprender la percepción de los adolescentes sobre el suicidio en una escuela secundaria en Sobral-Ceará, Brasil. Estudio exploratorio descriptivo con abordaje cualitativo realizado con 30 adolescentes, de 3 er año de bachillerato en una escuela del interior de Ceará, en septiembre de 2018. La investigación utilizó una entrevista semiestructurada con las siguientes preguntas: ¿Qué entiendes por ¿suicidio? ¿Puedes identificar una idea suicida? Si es así, describa. Si conocieras a alguien que estaría pensando en suicidarse, ¿qué harías para tratar de ayudarlo? El análisis de la información siguió los preceptos de Minayo (2010). Se evidenció que los adolescentes tienen conocimientos sobre el tema, así como algunas estrategias de apoyo ante el suicidio. La mayoría de los adolescentes informaron que su primer contacto con el tema del suicidio fue en la escuela, y que conocían o vivían con alguien que ya se había suicidado con él. Es de destacar que este trabajo puede animar tanto a los familiares como en el ámbito de la atención a orientar a los adolescentes sobre el suicidio y sobre la ruptura del tabú que tiene este tema. También refuerza la importancia de realizar más investigaciones sobre el tema, para ampliar nuevas estrategias de intervención con este grupo y las instituciones de salud y educación involucradas.

Palabras clave: Percepción del suicídio; Factores de riesgo; Prevención; Adolescentes.

\section{Introdução}

A fase da adolescência é um período que se posiciona, entre a fase da infância e a fase adulta, estende-se pela faixa etária de 10 a 19 anos (WHO, 2008). No entanto, o estatuto da criança e do adolescente (ECA) considera criança até os 12 anos incompletos e adolescente dos 12 aos 18 anos (Brasil, 1990).

É nessa fase da adolescência que ocorrem mudanças de ordem emocional que são de extrema importância para o indivíduo, tais como o desenvolvimento da autoestima e da autocrítica, questionamento dos valores dos pais e dos adultos em geral (Friedman, 1994). Todas essas mudanças podem desenvolver nesta fase processos de adoecimento e sofrimentos, entre eles, destaca-se neste estudo o suicídio.

O suicídio está entre as três principais causas de morte entre indivíduos entre 15 a 45 anos de idade. Segundo dados da Organização Mundial da Saúde (OMS) o suicídio é responsável por cerca de um milhão de mortes por ano, 1,4\% do total de mortes, e esses valores não incluem as tentativas de suicídio, que é de 10 a 20 vezes mais recorrente que o suicídio (WHO, 2014).

Assim, por possuir um coeficiente considerado baixo e por ter uma população aparentemente grande, o Brasil está entre os dez países que tem os maiores índices de suicídio segundo dados coletados pela OMS (WHO, 2014). Em 2011, houve 9.852 suicídios registrados oficialmente no Brasil, isso representava 27 suicídio por dia no país (Brasil, 2013).

Mesmo com números crescentes, discutir sobre o suicídio ou quais os fatores que levam o adolescente a atentar contra a própria vida, são assuntos, muitas vezes, não comentados pela sociedade (Barbosa, 2011).

Carvalho et al. (2013) traz que o suicídio na adolescência é um evento difícil e multifatorial que é entendido como um dos maiores problemas públicos enfrentados na atualidade, um problema não apenas nacional, mas mundial. Estima-se que em cerca de 45 segundos ocorre um suicídio, tendo uma média de 1.920 vidas ceifadas diariamente (WHO, 2014). Esse número eleva-se ainda mais se levado em consideração as somas causadas por homicídios, acidentes de transporte, conflitos e guerras (Varnik, 2012; WHO,2014).

Conforme Sadock (2007) existem vários fatores que podem levar um adolescente a tentar o suicídio, dentre eles podese citar o abuso sexual na infância ou negligência, histórico de doenças psiquiátricas na família ou algum tipo de perturbação 
psiquiátrica, sem nenhum apoio parental, problemas com a justiça, contatos com armas. Em sua pesquisa Sadock (2007) verificou que a tentativa de suicídio entre homens e mulheres, as mulheres realizam duas ou três vezes mais que os homens, no entanto eles são mais eficazes devido a recorrer a métodos altamente destrutivos. Já os adolescentes entram em conflito interno que não são mais crianças, porém não tem maturidade suficiente para tomar decisões adultas.

A adolescência é uma fase de desenvolvimento em que aspectos essenciais de uma pessoa são adquiridos. Nesse período, formam-se pilares essenciais da personalidade, como o sentimento de identidade própria e a progressiva discriminação entre o que é seu e o que liga o sujeito ao meio social.

Vieira e Coutinho (2008) investigaram a ocorrência de suicídio no meio acadêmico realizaram uma pesquisa com 233 estudantes do curso de Psicologia de uma universidade pública, o resultado apontou um índice significativo de depressão e ideação suicida. Salienta-se a pertinência de estudos que abordem não somente a temática suicídio, mas também sobre a percepção de todos sobre o tema, para ter subsídios que norteiem a tomada de decisão sobre programas de prevenção à saúde e desses agravos.

Reconhece-se que outro meio bastante eficiente de prevenção ao suicídio está em dialogar sobre o tema, pois, segundo a OMS, o estigma desse tipo de morte ainda tem efeitos devastadores sobre a população e ainda é considerado um grande tabu no meio social. Assim, a relevância deste estudo dá-se por trazer esse assunto para a discussão com a sociedade, bem como reconhecendo a percepção dos adolescentes acerca do suicídio, para, assim, realizar trabalhos de educação e promoção de saúde. Logo, questiona-se: qual a percepção dos adolescentes acerca do suicídio?

Diante do exposto, o presente estudo objetivou conhecer a percepção dos adolescentes acerca do suicídio em uma Escola de ensino médio em Sobral-CE Ceará, Brasil.

\section{Metodologia}

Esse estudo é do tipo exploratório descritivo com abordagem qualitativa. O presente estudo foi realizado em uma Escola de ensino médio em um Município do interior do Ceará, Brasil, em setembro de 2018.

Os participantes do estudo foram 30 adolescentes, do $3^{\circ}$ ano do ensino médio. Como critério de inclusão optou-se por adolescentes que tiveram contato com o tema, seja por rede social ou conhecimento presencial. A imersão dos pesquisadores junto ao grupo antecedeu 3 meses antes do apanhado de dados. Realizou-se 10 oficinas com uma turma de 50 adolescentes. As oficinas eram geridas para e com os adolescentes e, era debatido nas rodas de conversas temas que os adolescentes escolhiam, exceto suicídio, uma vez que, não era intenção dos pesquisadores influenciar previamente nas respostas do grupo. Essa decisão foi tomada com um objetivo primário, ou seja, vincular-se com o grupo e, a partir disso, conseguir extrair informações sustentáveis a pesquisa. Após, essa abordagem, foi questionado ao grupo participante quem já tinha contato com o tema. Dos 50 adolescentes, 40 relataram convivência direta ou indireta com a temática. Ao indagar sobre o aceite em participar da pesquisa, 30 aceitaram responder a entrevista semiestruturada com as seguintes perguntas: O que você entende por suicídio? Você consegue identificar uma ideia suicida? Se sim descreva. Se você conhecesse alguém que estaria pensando em cometer suicídio o que você faria para tentar ajuda-lo? A análise das informações seguiu os preceitos de Minayo (2010). A pesquisa seguiu os princípios éticos da Resolução nº 466, de 12 de dezembro de 2012 e tem o seguinte parecer: 2.971.455.

Entretanto, a princípio, se esclarece que para essa coleta de dados os adolescentes foram nomeados com a letra 'A' que representa a palavra adolescente e os números são a ordem em que cada adolescente foi entrevistado, assim os codinomes dos participantes ficaram: A1, A2, A3... A30. Com essas informações foi construída as áreas temáticas: conhecimento dos adolescentes acerca do suicídio e estratégias de apoio conhecida pelo adolescente diante do suicídio. 


\section{Resultados e Discussão}

\section{Conhecimento dos adolescentes acerca do suicídio}

Esta categoria tem como objetivo demonstrar o conhecimento dos adolescentes acerca do suicídio, tendo como base as respostas obtidas por 30 adolescentes. Nesse sentido a Figura 1 apresenta uma síntese destas informações.

Figura 1 - Conhecimento dos adolescentes acerca do suicídio. Viçosa do Ceará, Brasil, 2018.

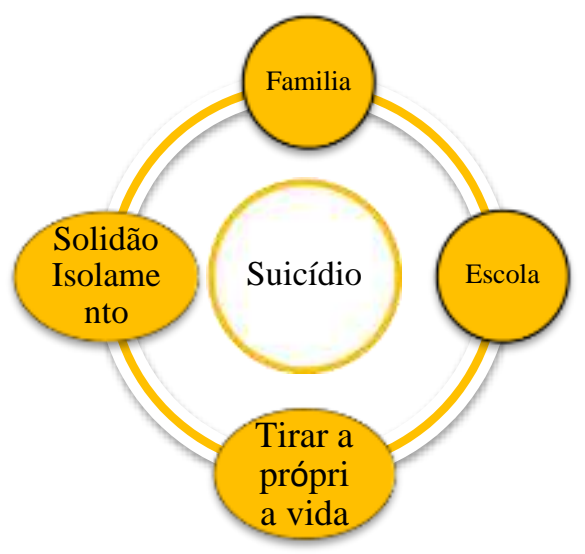

Fonte: Autores.

Constatou-se conforme na Figura 1 que os 30 entrevistados compreendem o significado de suicídio e conviveram com alguém que tentou o suicídio como pode ser confirmado nas falas abaixo;

Quando um ser humano tenta tirar a própria vida (A2).

Sim, retirar a própria vida. Meu primo. Tinha 15 anos e se enforcou (A17).

Quando uma pessoa se isola demais e começa a falar coisas sobre acabar com a própria vida. É a tal da depressão (A10).

Quando alguém retira a própria vida. Na escola é apresentado. Taxa de suicídio no país e os possíveis motivos (A28).

A depressão. Ela é cruel. Mata muitas pessoas (A12).

Já o termo suicídio foi registrado pela primeira vez no dicionário, Oxford English Dictionary, em 1661 na Inglaterra, como "o ato de matar a si mesmo intencionalmente", porém até 1755 a palavra não havia sido citada em nenhum outro dicionário inglês, o que demonstra um preconceito e um tabu envolvendo o ato suicida (Meleiro et al., 2004) é entendido como dar fim a própria vida espontaneamente (Schlosser, 2014).

Entre os adolescentes, a depressão é apontada como o distúrbio psicológico mais incidente e está relacionada com ideia de suicidio (Beesdo, Pine, Lieb e Wittchen, 2010). Em concordância temos, os transtornos de humor são os principais transtornos psiquiátricos associados ao suicídio, seguidos do uso e abuso de substâncias psicoativas (Brasil, 2006). Embora o suicídio seja algo complexo e multifatorial, alguns fatores de risco são comuns na maioria dos casos, entre os quais incluem-se a depressão, ela foi confirmada como forte preditor de comportamento que desperta o interesse pelo suicidio durante adolescência (Sheftall et al., 2014). A autoestima foi um aspecto mais especificamente associado ao engajamento em CS, 
relação que pode ser explicada no sentido de que a baixa autoestima pode ser um indicador da presença de transtorno depressivo, principal fator de risco suicida (Braga e Dell'Aglio, 2013).

A OMS (2012), relata que a presença pontual de ideias suicidárias constitui um processo normativo da adolescência, na medida em que pode estabelecer uma tentativa de resposta aos problemas existenciais característicos da adolescência. O comportamento suicida tem sido conceitualizado como um contínuo de pensamentos e comportamentos desde a ideação até o suicídio em si (Ganz, Braquehais e Sher, 2010). Esta questão coloca-se na medida em que poderá potenciar representações mentais mais negativas de si e dos outros que se associa, não raras vezes, com a ativação de mecanismos desadaptativos de gestão de problemas, nomeadamente comportamentos de destruição pessoal (Macedo, 2010).

Martins, Trindade e Almeida (2003), ao discutirem sobre fatores de proteção à depressão na adolescência, afirmam os adolescentes relatam precisar de liberdade para se divertir, informam sobre a necessidade de apoio e de proteção para sentiremse seguros. Os autores demonstram que, para seus sujeitos, o bem-estar está ligado ao sentimento de proteção oferecido pelos pais. Acrescentam ainda que o apoio e a proteção são elementos significativos para a vida e que a responsabilidade para seu futuro está, neste momento, colocada em agentes externos (pais, boa escola, entre outros).

Dutra (2002) considera que a solidão e um sentimento normal entre os adolescentes que tentam o suicídio, esses jovens argumentam sentir falta de ter amigos e relata não ter ninguém para compartilhar suas experiências e tristezas, com isso mostrando uma maior probabilidade de desenvolver problemas emocionais, comportamentais ou afetivos. Prieto e Tavares (2005) relataram que a falta de convivência com amigos durante a infância ou até mesmo na adolescência pode caracterizar como um fator de risco ao suicídio, pois troca afetivas nessas fases da vida reduzem os impactos das experiências adversas.

Sobre esta vivência depressiva, afirma que está se revela, comumente, através de sentimento de vazio, tédio, indiferença, solidão, abandono, impressão de ser mal-amado, incompreendido ou rejeitado. $\mathrm{O}$ autor acrescenta que os sinais da depressão podem ser "uma resposta inevitável" a um mundo sentido como frustrante (Dutra, 2001).

$\mathrm{Na}$ entrevista 19 adolescentes, dos 30 entrevistados falaram que o primeiro contato com o tema suicídio foi na escola. A escola tem papel estratégico para a promoção e proteção da saúde dos alunos, pois é o local onde são reproduzidos os padrões de comportamentos e relacionamentos que podem por em risco a saúde dos jovens. Nesse sentido, acredita-se que se a escola possa ser um local privilegiado para prevenir situações problemáticas, já que aspectos relacionados ao meio familiar, grupo de amigos e escola são de extrema importância para a saúde mental do adolescente (Beautrais, 2001).

A escola e constituída por um espaço privilegiado para a identificação da ideia suicida em adolescentes e que o professor tem um papel fundamental na identificação dos fatores de risco. Especificamente por que eles (professor) pode prestar assistência ao adolescente a entender possibilidades no existir assim vencendo seus sofrimentos. O professor representa um grande suporte ao adolescente. Uma vez formado sobre a temática, ele será capaz de identificar sinais de alerta em jovens com riscos suicidas, alguns desses sinais muitas vezes passam despercebidas pelos familiares (Silva, 2001).

90\% dos adolescentes entrevistados relataram que conheciam ou conviviam com algum indivíduo que já cometeu suicídio com isso, os riscos de conhecer ou conviver com indivíduos que tiraram a própria vida. A morte ocasionada pelo suicídio pode afetar diretamente o desenvolvimento do luto, fato que se deve por uma morte repentina e violenta, cercada de tabu e preconceitos e na maioria das vezes em circunstâncias de patologias psiquiátricas precedente e intensos problemas familiares (Silva, 2008). O é um tipo de morte com um impacto muito prejudicial e pode ser devastador para os familiares do indivíduo que comete suicídio. Os sobreviventes são essas pessoas quer perderam algum membro da família por suicídio ou tenta sido afetado de alguma maneira pela morte da pessoa que se matou. Esses sobreviventes podem apresentar um grande aumento de chance de cometer um suicídio (CFP, 2013). 
Quando perguntando aos adolescentes: 'você conhece alguém próximo que já cometeu suicídio, dos 30 adolescentes entrevistados, 27 disseram que já conviveram ou conhecem alguém que cometeu suicídio. Algumas falas reforçam essa afirmação.

Já sim. Meu primo (A4).

Já tive um tio. Ele tomou chumbinho (A25).

Minha irmã. 13 anos (A7).

Um conhecido meu. Vizinho. Usava droga (A 11).

O suicídio pode afetar diretamente a vida de um adolescente que convive com um indivíduo que já tentou tirar a própria vida, pois os adolescentes se deparam com várias situações novas e pressões sociais, favorecendo condições próprias para que apresentem variações de humor e mudanças expressivas no comportamento. Entretanto, alguns mais sensíveis e sentimentais, podem desenvolver quadros francamente depressivos com notáveis sintomas de descontentamento, confusão, solidão, incompreensão e atitudes de rebeldia. Esse quadro pode indicar depressão, ainda que os sentimentos de tristeza não sejam os mais evidentes. Todos os seres humanos estão familiarizados com as emoções de alegria e tristeza. "Os indivíduos saudáveis experimentam estas emoções de um modo previsível, geralmente uma resposta a um estímulo externo [...]. Estas emoções são vividas por indivíduos saudáveis até certo ponto e por um período de tempo apropriado à situação [...]. Os indivíduos que sofrem de depressão experimentam um grau profundo de tristeza aparentemente não relacionada com estímulos externos e por longo período". Por isso é muito comum esses adolescentes conhecer algum indivíduo que já cometeu suicídio (Ballone, 2003).

\section{Estratégias de apoio conhecida pelo adolescente diante do suicídio.}

Essa categoria tem como objetivo demonstrar as estratégias de apoio conhecida pelo adolescente diante do ato suicida. Na Figura 2 é demonstrado os conceitos chaves apreendidos nas falas dos 8 adolescentes entrevistados.

Figura 2 Estratégias de apoio conhecida pelo adolescente diante do suicídio,Viçosa do Ceará, Brasil, 2018.

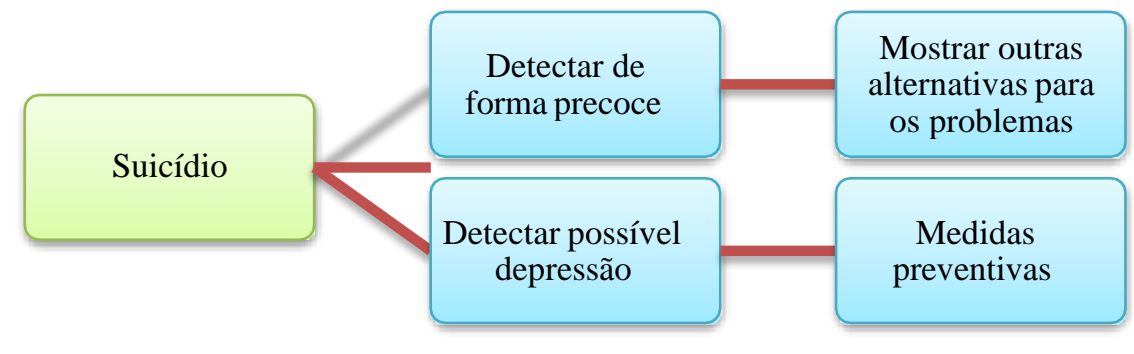

Fonte: Autores.

Como visto na figura 2 acima, os adolescentes têm consciência de estratégias de apoio diante do suicídio. Esses conceitos chaves são reforçados nas falas abaixo:

Temos que apoiar. O diálogo é importante (A21)

Já passei por isso. Converso. Tento mostrar outras alternativas. Que a vida é boa (A3). 
A família é importante. É uma saída (A 19).

Tem que ver se é depressão. Se for, tem que tratar. Se não, se mata (A12).

Acima de tudo. Tem que ter cuidado com a depressão. Notar se ela existe. E tratar (A30).

Levando em consideração a complexibilidade do problema do suicídio, sabendo da existência de uma parte da sociedade é construída de jovens e adolescentes que podem estar em grupos de riscos, é necessário unir forças para a realização de ações preventivas. Profissionais (médicos, psicólogos, terapeutas e professores) cujos saberes podem ser unidos precisam implementar estratégias conjunta assim aumentando o potencial das redes que existe na família, na escola e comunidade, o desafio da promoção do ato suicida consiste em perceber as situações que promovem a vulnerabilidade do adolescente e nas predisposições de intervenções eficazes. (OMS, 2000).

No que se refere às estratégias de minimizar o ato suicida, o Brasil avança em relação de criar políticas públicas que norteiam o desenvolvimento de intervenções em promoção da saúde. No ano de 2006, como Estratégia Nacional de Prevenção ao Suicídio, o País lançou o manual Prevenção de suicídio: manual voltado as equipes multiprofissionais de saúde mental, intuindo identificar de forma precoce condições que envolvem o suicídio na adolescência e dispor de estratégias preventivas. O manual destaca a importância das equipes da rede de atenção psicossocial nas estratégias de cuidado para minimizar o suicídio, descreve acerca dos fatores que predispõe a ideação e o ato suicida e aborda sobre o manejo das psicopatologias associadas ao risco de suicídio como a depressão, a esquizofrenia, a dependência de álcool ou drogas e os transtornos de personalidade. O manual também contribui descrevendo a abordagem correta de potenciais suicidas e as formas de ajudá-los. Além disso, menciona sobre o fluxo de pacientes e referência dos mesmos, quando estes estiverem sob risco de suicídio (Brasil, 2006).

O papel do enfermeiro nesse atendimento é acolher, mostrar e explicar ao paciente os serviços que possam lhe ajudar identificar comportamentos que favorecem o suicídio e fortalecer fatores de proteção, colaborando com a prevenção de tal comportamento. Para que a escuta terapêutica se realize com sucesso, é necessário que exista a comunicação entre paciente e profissional (Azevedo, Araújo e Vidal, 2015)

No entanto, quando os profissionais só dão atenção pontual aos casos, tratando sintomaticamente as lesões sem preocupação de contextualizar o evento, os atendimentos trazem pouca informação na identificação das vítimas, prejudicam o correto registro e não promovem uma possível prevenção de novos episódios (WHO, 2014).

No Brasil, embora se tenha conhecimento sobre as diretrizes nacionais para prevenção de suicídio, esse agravo recebe pouca atenção de políticas públicas de saúde, e há ausência de estratégias nacionais que debatam o assunto e proponham ações efetivas no sistema de saúde pública (Waiselfisz, 2014).

Alguns adolescentes argumentaram que uma das principais estratégias para combater o ato suícida e detectar uma ideação suicida de forma precoce. Para detectar um suicídio de forma precoce e necessário, ação multiprofissional, assim como para o comportamento suicida precisa-se de uma organização do trabalho baseado gestão de cuidado integrado, que requer intervenções técnicas e interação entre todos os membros da equipe de saúde, que, de maneira coordenada, colocam em pratica seus planos de ação. A comunicação, a articulação das ações e a compreensão das diferenças técnicas tornam-se indispensáveis para que os profissionais de saúde consigam alcançar seus objetivos. Portanto, a soma dos saberes das diversas profissões envolvidas no ato do cuidar colabora para que este fenômeno seja abordado de forma mais possível e que seja garantida a preservação da vida (Abreu, Lima, Kohlrausch e Soares, 2012).

E muito importante sempre mostrar para o adolescente que tem outras alternativas. A compreensão dos estudos mais recentes amplia o entendimento acerca dos fatores que predispõem ao comportamento suicida, indicando que, para que se possa atuar de maneira preventiva diante dos comportamentos suicidas, é preciso estar ciente e alerta para os diversos fatores que predispõe ao ato suicida e de proteção (Borges et al., 2008). 
Muitos adolescentes associam suicídio com depressão, por isso muitos falaram que uma boa estratégia para prevenir o suicídio seria detectar uma depressão o quanto antes. Encontramos que a prevalência de ideação suicida entre adolescentes é alta e está significativamente relacionada a depressão; consumo de álcool e a utilização de outras drogas; violência física; problemas de relacionamento com os pais; tristeza e solidão (Moreira e Bastos, 2015).

Quando associada à depressão, a ideação suicida pode ser prevenida, desde que o adolescente receba devido tratamento (Moreira e Bastos, 2015). Como fatores associados da ideação suicida entre adolescentes, encontramos (Souza et al., 2010): baixa escolaridade da mãe; baixa escolaridade do adolescente; sedentarismo etc. Nesta direção encontramos como aspectos da ideação suicida que distinguem um jovem saudável de um que se encontra em crise suicida (Souza, 2010) a intensidade desses pensamentos; a profundidade e a duração desses pensamentos; o contexto em que esses pensamentos surgem e a impossibilidade de se distanciar desse processo de pensamento.

\section{Considerações Finais}

Com o desenvolvimento desta pesquisa, contribuiu-se para o conhecimento da percepção dos adolescentes acerca do ato suicida. Este estudo evidenciou com essas informações, que os adolescentes possuem conhecimento sobre a temática, assim como dispõe de algumas estratégias de apoio diante do suicídio.

Evidencia-se que este trabalho possa estimular tanto os familiares, como no campo da assistência a orientar os adolescentes sobre esta discussões e sobre a quebra do tabu que esse tema possui. Reforça-se também a importância de realizar novas pesquisas sobre o tema, para ampliar novas estratégias de intervenções junto a este grupo, as Instituições de saúde e educação envolvidos.

Esse trabalho teve algumas limitações, como por exemplo, ter entrevistado apenas 30 adolescentes, tratando-se de uma realidade microrecortada, entretanto, os dados colhidos junto aos adolescentes forneceram informações relevantes para a pesquisa, uma vez que, se deu voz ao grupo que vêm sendo mais acometido pelo suicídio, isso torna este estudo relevante e atual sobre a temática.

\section{Referências}

Abreu, K. P., Lima, M. A. D. da S., Kohlrausch, E., \& Soares, J. F. (2010). Comportamento suicida: fatores de risco e intervenções preventivas. Revista Eletrônica De Enfermagem, 12(1). https://doi.org/10.5216/ree.v12i1.9537

Azevedo, A. L., Araújo, S. T. C., \& Vidal, V. L. L. (2015). Como o estudante de enfermagem percebe a comunicação com o paciente em saúde mental. Acta Paul Enferm. 28(2):125-131.

Ballone, G. J. (2003). Suicídio na adolescência. http://www.psiqweb.med.br/site/DefaultLimpo.aspx?area=NO/LerNoticia\&idNoticia=145

Barbosa, F. O., Macedo, P.C. \& Silveira, R. M. C (2011). Depressão e Suicídio. Rev. SBPH.14(1): 233-243.

Beautrais, A. L. (2001). Suicides and serious suicide attempts: two populations or one? Psychol Med., 31(5):837-845.

Beesdo, K., Pine, D.S., Lieb, R. \& Wittchen, H.U. (2010). Incidence and risk patterns of anxiety and depressive disorders and categ orization of generalized anxiety disorder. Archives of General Psychiatry, 67(1):47-57.

Borges, V. R., Werlang, B.S.G. \& Copatti M. (2008). Ideação suicida em adolescentes de 13 a 17 anos. Barbarói, 11(1):109-123.

Braga, L. L., \& Dell'aglio D. D. (2013). Suicídio na adolescência: fatores de risco, depressão e gênero. Contextos Clínicos; 6(1):2-14.

Brasil. Mistério da Saúde. Constituição da República Federativa do Brasil Lei 8.069, de 13 de Julho de 1990. (1990). Estatuto da Criança e do Adolescente. Brasília: Ministério da Justiça.

Brasil. Mistério da Saúde. Constituição da República Federativa do Brasil. Ministério da Saúde. (2006) Prevenção do suicídio: manual dirigido a profissionais das equipes de saúde mental.

Brasil. Mistério da Saúde Constituição da República Federativa do Brasil (2013). Estatísticas vitais, mortalidade.

Carvalho, Á., Peixoto, B., \& Saraiva C. B. (2011). Plano Nacional de Prevenção de Suicídio Direção-Geral da Saúde Programa Nacional para a Saúde Mental Plano. 
Research, Society and Development, v. 10, n. 13, e313101319217, 2021

(CC BY 4.0) | ISSN 2525-3409 | DOI: http://dx.doi.org/10.33448/rsd-v10i13.19217

Conselho Federal de Psicologia (2013). O Suicídio e os desafios para a psicologia. Brasília.

Dutra, E. (2012). Suicídio de universitários: o vazio existencial de jovens na contemporaneidade. Estud. pesqui. psicol., Rio de Janeiro, 12 (3): $924-937$.

Friedman, H. L. (1994). Th e promotion of adolescent health: principles of eff ective intervention. Mexico: Latin American and Caribbean Meeting on Adolescent Health. Mimeo.

Ganz, D., Braquehais, M.D., \& Sher, L. (2010). Secondary prevention of suicide. PLoS Medicine, 7(6).

Macedo, M. M. K. (2010). Adolescência e psicanálise: intersecções possíveis (2a ed.). Porto Alegre: Edipucrs.

Martins, P. O., Trindade, Z. A., \& Almeida, A. M. O. (2003). O ter e o ser: representações sociais da adolescência entre adolescentes de inserção urbana e rural. Psicol. Reflex. Crit., Porto Alegre 16(3):555-568.

Meleiro, A. M. A. S, \& Bahls, S. C. O. (2004). O comporto suicida. In: Meleiro, A. M. A. S; Teng, C. T; Wang, Y. P. Suicídio: Estudos Fundamentais. p. 13 16. São Paulo. Segmento Farma.

Minayo, M. C. S., Meneghel, S. N., \& Cavalcante, F. G. (2012). Suicídio de homens idosos no Brasil. Ciênc. saúde coletiva [online], 17(10):2665-74.

Moreira, L. C. O., \& Bastos, P. R. H. O. (2015). Prevalência e fatores associados à ideação suicida na adolescência: revisão de literatura. Psicologia Escolar e Educacional, [s.1.], 19(3):445-453.

Prieto, D., \& Tavares, M. (2005). Fatores de risco para suicídio e tentativa de suicídio: incidência, eventos estressores e transtornos mentais. J Bras Psiquiatr., 54(2:146-154.

Sadock, B. J., Sadock, V. A. (2007). Kaplan \& Sadock compêndio de psiquiatria: ciência do comportamento e psiquiatria clínica. 9. ed. Porto Alegre: Artmed, p. 1364-1403

Schlosser, A. (2014). Interface entre saúde mental e relacionamento amoroso: um olhar a partir da psicologia positiva. Pensando fam. [online], 18 (2):17-33.

Sheftall, A. H., Mathias, C. W., Furr, R. M. \& Dougherty, D. M. (2013). Adolescent attachment security, family functioning, and suicide attempts. Attachment \& Human Development, 15(4):368-383.

Silva, T. C. M. F. (2007). A escola como espaço de prevenção ao suicídio de adolescentes - relato de experiência. Revista Inter Ação, [S.1.], 27(1): 99-114.

Silva, D. R. (2008). Luto disfuncional: contribuições e intervenções da terapia familiar sistêmica. In: MACEDO, R. M. S. (Org.). Terapia familiar no Brasil na última década. São Paulo: Roca, p. 562-571.

Souza, M. L. P., Deslandes, S. F. \& Garnelo, L. (2010). Modos de vida e modos de beber de jovens indígenas em um contexto de transformações. Ciência Saúde Coletiva, 15(3): 709-16.

Värnik, P. (2012). Suicide in the world. International Journal of Environmental Research and Public Health, 9(1):760- 771.

Vieira, K. F. L., \& Coutinho, M. P. L. (2008). Representações sociais da depressão e do suicídio elaboradas por estudantes de psicologia. Psicologia: Ciência e Profissã, 28(4):714-27.

Waiselfisz, J. J. Mapa da Violência. (2014). Os Jovens do Brasil.

World Health Organization (2014). Preventing suicide: a global imperative Geneva: WHO

World Health Organization (2002). World report on violence and health Geneva: WHO.

World Health Organization (2008). Adolescent health. WHO. 